\title{
OEM perspective on decoupled steering systems
}

Riccardo Ficca, Jaguar Land Rover Limited, UK

This manuscript is not available according to publishing restriction. Thank you for your understanding. 